\title{
Macrolides, mucoactive drugs and adherence for the management of bronchiectasis
}

\author{
To the Editor:
}

The publication of the first European Respiratory Society guidelines for management of bronchiectasis [1] is a landmark of recent advancement in the field. Whilst the British Thoracic Society guidelines [2] delineated mechanisms of pathogenesis and offered recommendations for management of bronchiectasis, the latest document specifically addressed nine clinically important questions that may better inform clinical decisions. Although it is likely that these recommendations can be extrapolated to patients in Asian countries such as China, we have some further suggestions and/or recommendations for refinement.

First, the optimal length of macrolide administration should be clarified. No clear definition of long-term administration (e.g. 6, 12 or 24 months) was stated in the guidelines. Although gastrointestinal tract (e.g. nausea and diarrhoea), cardiac (e.g. prolonged QT intervals) and hearing disorders have been reported, most adverse events were mild, and can be readily identified and managed. The major issue concerning long-term use of macrolides lies within clinical benefit. Despite convincing positive outcomes (significantly reduced exacerbation frequency and improved quality of life) [3], the observational duration in most clinical trials [4-6] was <2 years. Notably, prolonged use of macrolides has been associated with changes in sputum microbial compositions (determined with $16 \mathrm{~S}$ ribosomal RNA sequencing) and an increased risk of harbouring macrolide-tolerant Pseudomonas aeruginosa [7]. Furthermore, long-term use of macrolides did not reduce exacerbation frequency among patients from whom $P$. aeruginosa was not isolated [7], questioning the validity of long-term prescription in bronchiectasis. Whilst long-term macrolides should be prescribed to patients in whom oral antibiotics are contraindicated, no clinical trials to date have directly addressed the long-term benefits of macrolides for bronchiectasis patients with and without $P$. aeruginosa colonisation. It remains unclear whether prolonged use of macrolides (e.g. $>2$ years) could reduce the risks of bronchiectasis exacerbations. How long should the optimal duration be for macrolides prescription needs to be addressed. Caution should be exercised when interpreting findings from existing clinical trials.

Second, indications for use of mucoactive drugs should be better defined. Airway clearance, including upper airway clearance (e.g. nasal irrigation for patients with chronic rhinosinusitis), and facilitation of sputum expectoration are cardinal management recommendations for bronchiectasis. No existing trials have confirmed the superiority of airway clearance and mucoactive drugs. Physicians are concerned about which subpopulation might benefit more from mucoactive drugs. The recommendation to prescribe mucoactive drugs in patients who have difficulty in expectoration despite maintenance airway clearance was primarily based on mixed findings from clinical trials, which had limited sample sizes and durations. Whereas effects of inhaled mannitol on clearing sputum and prolonging the time to exacerbations were convincing, no trials have investigated whether other mucoactive drugs (high-dose $\mathrm{N}$-acetylcysteine, carbocisteine and ambroxol) provide clinical benefits in bronchiectasis. Apart from mucolytic effects, mucoactive drugs (particularly at higher doses) confer anti-inflammatory and antioxidative stress effects that clinically translate into reduced exacerbation frequency (particularly repetitive exacerbations), longer time to exacerbation and improved quality of life in chronic obstructive pulmonary disease [8, 9]. Therefore, higher doses of mucoactive drugs might confer similar clinical benefits in bronchiectasis. Furthermore, the optimal duration of mucoactive drug administration needs to be determined. The current guidelines mostly focused on inhaled mucoactive drugs; however, whether oral mucoactive drugs elicit

@ERSpublications

Macrolides, mucoactive drugs and adherence are crucial for the management of bronchiectasis http://ow.ly/H4oq30fZArC

Cite this article as: Guan W-jie, Huang Y, Chen C-lan, et al. Macrolides, mucoactive drugs and adherence for the management of bronchiectasis. Eur Respir J 2018; 51: 1701987 [https://doi.org/10.1183/ 13993003.01987-2017]. 
fewer side-effects and achieve better outcomes remains an open question. In light of the fact that airway clearance alone might not be sufficient for maintenance therapy and that self-administered airway clearance has not been regularly performed by bronchiectasis patients, we suggest that mucoactive drugs be used regardless of whether airway clearance is applied.

Finally, the importance of adherence to maintenance treatment has not been properly addressed. According to findings from our patients' interview, only $\sim 40 \%$ of patients were adherent to maintenance therapy, defined as continuous use of at least one category of medication and/or airway clearance technique (unpublished data). Patients who were adherent to therapy commented that withdrawal of medications would readily lead to progressive worsening of respiratory symptoms, whereas patients who were not adherent to maintenance therapy asserted that treatment resulted in very limited clinical benefits, that they already have "got used to daily coughing and sputum expectoration" and that prolonged use of medications might lead to "irreversible adverse effects" (e.g. frailty, infertility and gastrointestinal/renal disorders). This echoed the findings of McCullough et al. [10], who demonstrated that beliefs about treatment, perceived treatment burden, the number of prescribed medications and age were predictors of adherence. Despite limited evidence supporting the benefits of long-term therapy, we did notice that nonadherence or complete withdrawal of medication might predispose to poor clinical outcomes, such as radiological progression and/or worsening of respiratory symptoms. More studies are warranted to determine the consequences of medication withdrawal (e.g. clinical deterioration), particularly following a longer period of time. Results from these studies would shed light on the critical roles of maintenance therapy in bronchiectasis. Refinement of guidelines (incorporating recommendations to strengthen patient education) may achieve better outcomes.

Wei-jie Guan ${ }^{1,2}$, Yan Huang ${ }^{1}$, Chun-lan Chen ${ }^{1}$, Rong-chang Chen ${ }^{1}$ and Nan-shan Zhong ${ }^{1}$

${ }^{1}$ State Key Laboratory of Respiratory Disease, National Clinical Research Center for Respiratory Disease, Guangzhou Institute of Respiratory Disease First Affiliated Hospital of Guangzhou Medical University, Guangzhou Medical University, Guangzhou, China. ${ }^{2}$ Sino-French Hoffmann Institute, Guangzhou Medical University, Guangzhou, China.

Correspondence: Rong-chang Chen, State Key Laboratory of Respiratory Disease, National Clinical Research Center for Respiratory Disease, Guangzhou Institute of Respiratory Disease, First Affiliated Hospital of Guangzhou Medical University, 151 Yanjiang Road, Guangzhou, Guangdong, China. E-mail: chenrc@vip.163.com

Received: Sept 282017 | Accepted after revision: Sept 302017

Support statement: This work was supported by National Natural Science Foundation grant number 81400010, Pearl River S\&T Nova Program of Guangzhou grant number 201710010097, Guangdong Province Universities and Colleges Pearl River Scholar Funded Scheme 2017 (to W-J. Guan), Changjiang Scholars and Innovative Research Team in University grant ITR0961, The National Key Technology R\&D Program of the 12th National Five-year Development Plan grant 2012BAI05B01 and National Key Scientific \& Technology Support Program "Collaborative innovation of Clinical Research for chronic obstructive pulmonary disease and lung cancer" grant number 2013BAI09B09 (to N-S. Zhong and R-C. Chen). Funding information for this article has been deposited with the Crossref Funder Registry.

Conflict of interest: Disclosures can be found alongside this article at erj.ersjournals.com

Acknowledgements: W-J. Guan, Y. Huang and C-L. Chen drafted the manuscript; R-C. Chen and N-S. Zhang critically reviewed the manuscript and approved final submission.

\section{References}

1 Polverino E, Goeminne PC, Mcdonnell MJ, et al. European Respiratory Society guidelines for the management of bronchiectasis. Eur Respir J 2017; 50: 1700629.

2 Pasteur MC, Bilton D, Hill AT. British Thoracic Society guidelines for non-CF bronchiectasis. Thorax 2010; 65: i1-i58.

3 Gao YH, Guan WJ, Xu G, et al. Macrolide therapy in adults and children with non-cystic fibrosis bronchiectasis: a systematic review and meta-analysis. Plos One 2014; 9: e90047.

4 Wong C, Jayaram L, Karalus N, et al. Azithromycin for prevention of exacerbations in non-cystic fibrosis bronchiectasis (EMBRACE): a randomised, double-blind, placebo-controlled trial. Lancet 2012; 380: 660-667.

5 Altenburg J, de Graaff CS, Stienstra Y, et al. Effect of azithromycin maintenance treatment on infectious exacerbations among patients with non-cystic fibrosis bronchiectasis: the BAT randomized controlled trial. JAMA 2013; 309: 1251-1259.

6 Serisier DJ, Martin ML, McGuckin MA, et al. Effect of long-term, low-dose erythromycin on pulmonary exacerbations among patients with non-cystic fibrosis bronchiectasis: the BLESS randomized controlled trial. JAMA 2013; 309: 1260-1267.

7 Rogers GB, Bruce KD, Martin ML, et al. The effect of long-term macrolide treatment on respiratory microbiota composition in non-cystic fibrosis bronchiectasis: an analysis from the randomised, double-blind, placebo-controlled BLESS trial. Lancet Respir Med 2014; 2: 988-996.

8 Zheng JP, Kang J, Huang SG, et al. Effect of carbocisteine on acute exacerbation of chronic obstructive pulmonary disease (PEACE Study): a randomised placebo-controlled study. Lancet 2008; 371: 2013-2018. 
9 Zheng JP, Wen FQ, Bai CX, et al. Twice daily $N$-acetylcysteine $600 \mathrm{mg}$ for exacerbations of chronic obstructive pulmonary disease (PANTHEON): a randomised, double-blind placebo-controlled trial. Lancet Respir Med 2014; 2: $187-194$

10 McCullough AR, Tunney MM, Stuart Elborn J, et al. Predictors of adherence to treatment in bronchiectasis. Respir Med 2015; 109: 838-845.

Copyright @ERS 2018

From the authors:

We thank W-J. Guan and colleagues for their comments on the European Respiratory Society (ERS) guidelines for bronchiectasis [1]. We agree these are an important landmark for the field, and hope that clinicians, health professionals, patients and researchers will use the guidelines to improve the quality of care for bronchiectasis patients and to use the gaps identified to stimulate further research $[1,2]$.

We did not address the optimal duration of therapy with macrolides because there are no data to determine for how long patients should be treated [3-5]. Since the majority of high-quality evidence derived from studies of 6-12 months, a pragmatic approach is to undertake a trial of treatment for 612 months, discontinuing if it is ineffective [3-6]. Statements such as these cannot be included as formal recommendations within ERS guidelines presently because of the rigour of the GRADE (Grading of Recommendations Assessment, Development and Evaluation) methodology. A PICO (patient, problem or population; intervention; comparison, control or comparator; and outcome) question, a systematic review and meta-analysis, and a grading of evidence are required to support specific statements in ERS guidelines. Until more evidence is available, there are insufficient data to make an evidence-based recommendation on macrolide duration [1].

Some of W-J. Guan and colleagues' statements are inaccurate. Macrolides were associated in one study with a change in microbiota composition using $16 \mathrm{~S}$ ribosomal RNA (rRNA) PCR and sequencing, but this is not the same as indicating "an increased risk of harbouring macrolide-tolerant Pseudomonas aeruginosa". Relative abundance by $16 \mathrm{~S}$ rRNA sequencing is not the same as bacterial load and none of the individuals with an increase in relative abundance of $P$. aeruginosa in this study became culture positive for $P$. aeruginosa [7]. The significance of changes in the microbiome are currently unknown and results from small experimental studies need to be interpreted with care [7].

The statement that macrolides do not reduce exacerbation frequency in patients not infected with $P$. aeruginosa is also incorrect. This conclusion is based on a subgroup analysis of 41 participants in the BLESS trial, which did not achieve statistical significance [4]. In making our recommendation that macrolides should be used as first-line prophylactic antibiotic therapy for patients without $P$. aeruginosa infection, we considered the totality of evidence supporting macrolide use [1, 3-5]. Macrolides significantly reduced exacerbation frequency in both the EMBRACE and BAT trials in populations that had relatively few patients with $P$. aeruginosa $[3,5]$. Our conclusions that macrolides reduce exacerbations in patients without $P$. aeruginosa infection and a history of exacerbations are based firmly on the available evidence [1].

We caution against extrapolating the results of the PEACE trial and similar studies in COPD to bronchiectasis. The extrapolation of results from studies in COPD and cystic fibrosis is problematic, as treatments such as recombinant human DNase and mannitol in cystic fibrosis and inhaled corticosteroids in COPD have not shown clear efficacy in bronchiectasis studies [1]. Mucolytics such as cysteine derivatives are widely used but we are unable to recommend them in an evidence-based guideline in the absence of trial data. W-J. Guan and colleagues suggest that these drugs may be used in patients not practicing airway clearance techniques. We disagree, and stand by our original evidence-based recommendation to use mucoactive drugs in patients practicing chest physiotherapy-based airway clearance [1]. While there is no evidence to support the use of cysteine derivatives, we found a number of

@ERSpublications

Only evidence-based recommendations were included in the @ERStalk bronchiectasis guidelines http://ow.ly/Vecb30g1gJy

Cite this article as: Chalmers JD, Polverino E. Macrolides, mucoactive drugs and adherence for the management of bronchiectasis. Eur Respir J 2018; 51: 1702033 [https://doi.org/10.1183/13993003.020332017]. 
small trials supporting the effectiveness of airway clearance $[8,9]$. While further research in this area is needed, as a nonpharmacological intervention, the panel concluded that airway clearance should be the first-line intervention in bronchiectasis.

Finally, we agree that it is important to optimise adherence to all therapies in bronchiectasis. Patients with bronchiectasis often have multiple comorbidities and have to take large numbers of medications [10]. We agree that patients are more likely to adhere to therapies that are effective and so we would advocate following the evidence-based recommendations put forward in the ERS guidelines for management of adult bronchiectasis. Minimising medication burden in mild disease reduces the likelihood of nonadherence and, hence, we advocate prophylactic antibiotic therapies only for patients with frequent exacerbations (three or more per year) and only advocate the introduction of mucoactive drugs where simple measures have failed to control symptoms. Such a stepwise approach is consistent with the principals of optimising adherence, and should be accompanied by patient education regarding the goals of therapy and the importance of treatment adherence.

James D. Chalmers ${ }^{1}$ and Eva Polverino ${ }^{2}$ on behalf of the European Respiratory Society Bronchiectasis Guidelines Task Force

${ }^{1}$ Scottish Centre for Respiratory Research, University of Dundee, Ninewells Hospital and Medical School, Dundee, UK.

${ }^{2}$ Vall d'Hebron Institut de Recerca, Barcelona, Spain.

Correspondence: James D. Chalmers, Scottish Centre for Respiratory Research, University of Dundee, Ninewells Hospital and Medical School, Dundee, DD1 9SY, UK. E-mail: jchalmers@dundee.ac.uk

Received: Oct 042017 | Accepted: Oct 052017

Conflict of interest: Disclosures can be found alongside this article at erj.ersjournals.com

\section{References}

1 Polverino E, Goeminne PC, McDonnell MJ, et al. European Respiratory Society guidelines for the management of bronchiectasis. Eur Respir J 2017; 50: 1700629.

2 Aliberti S, Masefield S, Polverino E, et al. Research priorities in bronchiectasis: a consensus statement from the EMBARC Clinical Research Collaboration. Eur Respir J 2016; 48: 632-647.

3 Wong C, Jayaram L, Karalus N, et al. Azithromycin for prevention of exacerbations in non-cystic fibrosis bronchiectasis (EMBRACE): A randomised, double-blind, placebo-controlled trial. Lancet 2012; 380: 660-667.

4 Serisier DJ, Bilton D, De Soyza A, et al. Inhaled, dual release liposomal ciprofloxacin in non-cystic fibrosis bronchiectasis (ORBIT-2): a randomised, double-blind, placebo-controlled trial. Thorax 2013; 68: 812-817.

5 Altenburg J. Effect of azithromycin maintenance treatment on infectious exacerbations among patients with non-cystic fibrosis bronchiectasis. JAMA 2013; 309: 1251-1259.

6 Chalmers JD, Aliberti S, Blasi F. Management of bronchiectasis in adults. Eur Respir J 2015; 45: 1446-1462.

7 Rogers GB, Bruce KD, Martin ML, et al. The effect of long-term macrolide treatment on respiratory microbiota composition in non-cystic fibrosis bronchiectasis: an analysis from the randomised, double-blind, placebo-controlled BLESS trial. Lancet Respir Med 2014; 2: 988-996.

8 Patterson JE, Bradley JM, Hewitt O, et al. Airway clearance in bronchiectasis: a randomized crossover trial of active cycle of breathing techniques versus Acapella. Respiration 2005; 72: 239-242.

9 Murray MP, Pentland JL, Hill AT. A randomised crossover trial of chest physiotherapy in non-cystic fibrosis bronchiectasis. Eur Respir J 2009; 34: 1086-1092.

10 McDonnell MJ, Aliberti S, Goeminne PC, et al. Comorbidities and the risk of mortality in patients with bronchiectasis: an international multicentre cohort study. Lancet Respir Med 2016; 4: 969-979. 